\title{
Assessment of Retinal Vascular Plexuses and Choriocapillaris in Adult High Myopia Using Enhanced Depth Imaging Optical Coherence Tomography Angiography
}

\author{
Mohamed Anwar El-Massry, Hassan Metwaly Bayoumy, Atef Hassan Sayed Abdellatif* \\ Ophthalmology Department, Faculty of Medicine, Al-Azhar University \\ *Corresponding author: Atef Hassan Sayed Abdellatif, E-mail: atefhassan95@gmail.com, Mobile: (+20) 01272229060
}

\begin{abstract}
Background: High myopia is a common eye health problem due to its sight threatening complications. Using the newly introduced noninvasive devices, it's easier now to assess the morphological, anatomical and pathological changes accompanied with high myopia.

Objectives: The aim of this study was to assess retinal vascular plexuses and choriocapillaris density and measuring the FAZ in the SCP \& DCP with also assessment of central macular and subfoveal choroidal thickness in high myopia in adults using enhanced depth imaging optical coherence tomography angiography. Patients and Methods: This prospective observational study was carried out from June 2018 to November 2018 on 75 eyes of patients attending outpatient clinic of Ophthalmology department of Research Institute of Ophthalmology in Giza.

Results: The best corrective visual acuity in the normal group was statistically significantly higher than the Myope group. The Macular thickness in the normal group was statistically significantly higher than the Myope group. The choroidal thickness in the normal group was statistically significantly higher than the Myope group. The FAZ DCP in the Myope group was statistically significantly higher than the normal group.

Conclusion: The OCTA is certainly able to take over other invasive imaging techniques and new researches using this device might be able to study different pathologies affecting the posterior segment.
\end{abstract}

Keywords: High myopia, OCTA, DCP

\section{INTRODUCTION}

High myopia is associated with a refractive error of at least $6 \mathrm{D}$ and/or axial length greater than $25.5 \mathrm{~mm}$. The latter leads to mechanical stretching and rarefaction of the retina and choroid resulting in various complications such as peripheral retinal degeneration, retinal tears, posterior staphyloma, lacquer cracks and choroidal neovascularisation ${ }^{(1)}$. In addition to the previously mentioned complications, affection of retinal and choroidal circulation due to decreased vessels density and flow will lead to defective nourishment of the posterior pole ${ }^{(2)}$.

Fundus Fluorescein Angiography and Indocynine Green have long been regarded the benchmark imaging modalities for evaluation of high myopic eyes. They provide two dimensional image sets that allow for dynamic visualisation of blood flow with a wide field of view. However, some major drawbacks for these 2 types of investigations will limit their usage in studying retinal and choroidal vascular changes in high myopic patients $^{(3)}$.

Firstly, achieving a detailed image of the deep retinal capillary plexus (DCP) and the choriocapillaris with these imaging techniques is not possible due to scattering of light by nerve fibre layer and in case of FFA; leakage of fluorescein dye that obscures detailed visualisation of deeper layers. Secondly, these are invasive techniques, using intravenous dye to obtain images which make them time consuming and annoying for the patients undergoing this procedure for follow up (4,5). Moreover, nausea and allergic reactions may develop due to repetitive usage of the dye while indocynine green is contraindicated in pregnancy and patients with kidneys disease ${ }^{(6)}$.

The recently introduced OCTA incorporated in EDI-OCT provided many implements that revolutionized retinal imaging. OCTA is based on the split-spectrum amplitude-decorrelation angiography (SSADA) algorithm and Segmentation algorithm ${ }^{(7)}$.

The former is based on detecting the reflectance amplitude variation of blood flow over time to distinguish vessels from static tissue while the latter produces transversal slices of the retinal and choroidal layer at any depth ${ }^{(11)}$.

Acquired images are then projected as separate en-face image for each layer for evaluation. Compared to the above mentioned techniques, it's a non invasive procedure using motion contrast instead of IV dye, making it more reliable and hazard-free for follow up patients with no fears of adverse effects or contraindications. Also it is less time consuming as, beside the fact that it is non invasive, it takes few seconds to obtain images for the vascular plexuses ${ }^{(\mathbf{1 2})}$.

The superficial vascular network is composed of long, horizontal arterioles and venules, 
originating from the superior and inferior arcades around the foveal avascular zone. An arteriole is easily differentiated from a venule because the arterioles are surrounded by a wider capillary-free zone. Arterioles and venules are connected by transverse capillaries, forming an interconnected plexus ${ }^{(12)}$.

The deep capillary plexus, unlike the superficial capillary plexus, was organized into capillary vortexes, that is, radial convergence of capillaries toward an epicentre.

The limits of each capillary vortex unit had a polygonal shape. Vertical anastomoses are present between the DCP and the SCP as small, slanted, interconnected anastomoses between the superficial and deeper vessels but with no explicit mention to venous drainage ${ }^{(13)}$.

The two capillary plexuses have a different pattern. In the SCP, capillaries are arranged as transverse capillaries forming an interconnected plexus between arterioles and venules. In the DCP, the pattern is completely different: the DCP is composed of polygonal units, in which the capillaries converged radially toward an epicenter that is called capillary vortex ${ }^{(14)}$.

\section{AIM OF WORK}

The aim of this study is to assess retinal vascular plexuses and choriocapillaris density and measuring the FAZ in the SCP \& DCP with also assessment of central macular and subfoveal choroidal thickness in high myopia in adults using enhanced depth imaging optical coherence tomography angiography.

\section{PATIENTS AND METHODS}

This prospective observational study was carried out from June 2018 to November 2018 on 75 eyes of patients attending outpatient clinic of Ophthalmology department of Research Institute of Ophthalmology in Giza.

\section{Board of Al-Azhar University.}

The study was approved by the Ethics

Patients were divided into two groups:

Group 1: 35 eyes of 18 patients with high myopia ranging from -6 to $-25 \mathrm{D}$.

Group 2: 40 normal healthy emmetrope eyes as a control group.

\section{Inclusion criteria}

o groups of patients were included in this study; a control group composed of 20 normal individuals with no significant error of refraction were compared to a study group of 20 patients with high myopia more than - 6D.

\section{Exclusion criteria}

Patients with any retinal or choroidal disease that might affect retinal vascular layers and/or the choriocapillaris were excluded. Also the presence of media opacities affecting quality of EDI OCT images as: corneal opacities, dense cataract and vitreous haemorrhage were excluded.

\section{All study participants were submitted to:}

1. Careful history taking to detect:

- Age of the patient.

- Mode of trauma if any.

- Previous ocular surgery (nature, date and place).

2. Full ophthalmological examination included:

A. BCVA measurement using Snellen's chart with conversion to log. MAR notation for statistical analysis.

B. Anterior segment examination using slit lamp biomicroscopy:

- Assessment of media clarity (cornea and crystalline lens i.e. no corneal opacity or cataract).

- Excluding anterior segment diseases or surgeries that could affect study results.

C. Intraocular pressure (IOP) measurement using Goldmann applanation tonometer.

D. Posterior segment examination using binocular indirect ophthalmoscope and indirect slit lamp biomicroscopy (+90 volk lens) for detailed evaluation of macula to detect:

- Preexisting macular pathology as diabetic retinopathy or macular hole.

- Retinal detachment.

- Retinal features of pathological myopia.

\section{Image Acquisition:}

OCT:

Standard Optical coherence tomography of the macula was done using (Heidelberg engineering, OCT spectralis, Germany).

This SD-OCT with "OCT2 Module" can record up to 85,000 A-scans per second with transverse section 6 um resolution.

TruTrack Active Eye Tracking is a feature enable this platform simultaneously images the eye with two beams of light.

One beam captures an image of the retina and maps over 1,000 points to track eye movement.

Using the mapped image as a reference, the second beam is directed to the desired location despite blinks or saccadic eye movements. TruTrack 
dual beam technology mitigates eye motion artifact and ensures point to-point correlations between OCT and Confocal Scanning Laser Ophthalmoscope (cSLO) images without post-processing of the data. The following imaging protocol was applied:

\section{- Macula radial:}

To detect single point central foveal thickness (SCFT) and any anatomical abnormalities.

The center of the radial scanning line was adjusted on the fovea identified by both the steepest foveal excavation and the highest foveal bulge (if visible). The protocol consisted of a sequence of 6 radial sections covering central $15^{\circ}$ recorded in the high-resolution mode (768 A-scans) with a spaced by $30.0^{\circ}$ between individual sections.

\section{- Macular thickness map:}

For measurement of Central Macular Thickness CMT among all retinal Layers, the central scanning line was on the fovea. The protocol consisted of a sequence of 19 horizontal sections covering central $15^{\circ}$ recorded in the highresolution mode (768 A-scans) with a distance of approximately 240 um between individual sections. These measurements were calculated automatically by the software in all retinal Layers. The central macular thickness was measured from inner border of the (ILM) to the inner border of the RPE (figure $1)$.

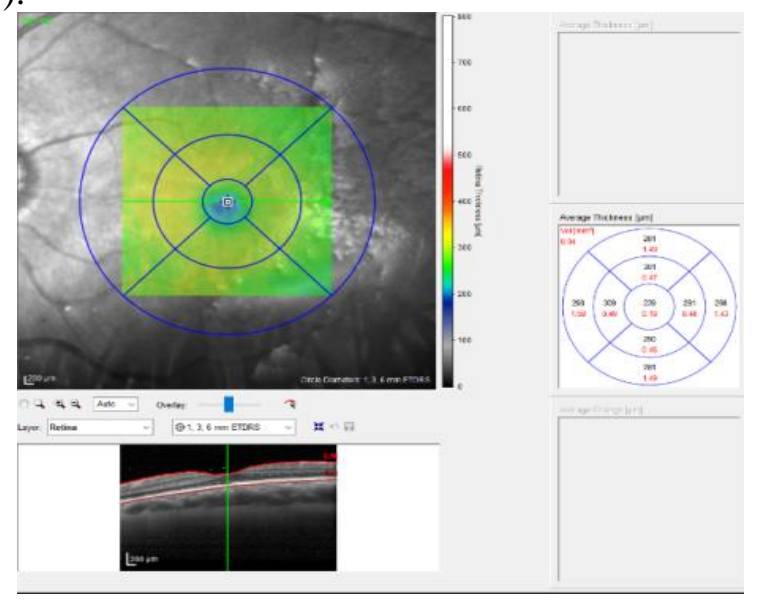

Figure 1: Shows OCT macular thickness map of all retinal layers measured from inner border of the

(ILM) to the inner border of the RPE.

While the subfoveal choroidal thickness was measured accurately from just below the RPE to the sclera. In cases where the software had difficulty recognizing the landmarks, the operator was able to manually correct the automatically generated borders.

\section{OCTA:}

Optical coherence tomographyangiography of the macula using (Heidelberg engineering, OCT spectralis, Germany).

\section{The following protocol was applied:}

OCT angiograms of the superficial and deep networks and choriocappilaris were captured. SCP, DCP and CC were distinctly evaluated using the automatic layers' segmentation done by OCTA, whereas the SCP was segmented from the ILM to INL, and the DCP was segmented from the INL to OPL. FAZ area was measured in both SCP and DCP using software "Draw region" tool to outline FAZ area (inner border of the most visible central blood capillaries) manually and Software Automatically calculate the outlined area.

The protocol consisted of a sequence of 256 sections covering central $3 \mathrm{~mm}^{2} \times 3 \mathrm{~mm}^{2}$ recorded in the high-resolution mode (512 A-scans) spaced by 6 $\mu \mathrm{m}$ between individual sections.

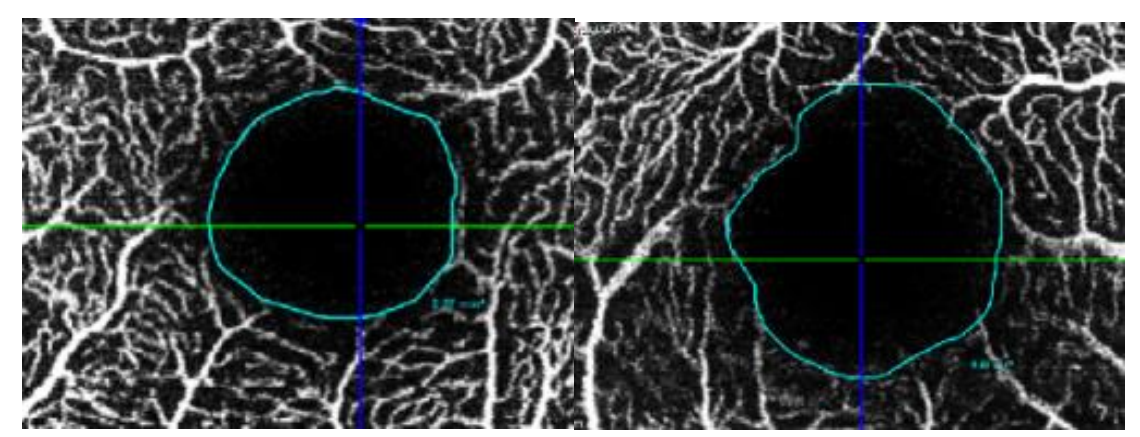

Figure 2: Measuring FAZ in SCP (left image) and DCP (right image). 
The results obtained were tabulated \& statistically analyzed using specific analytical program.

The study was conducted in accordance with the ethical standards stated in the Faculty of Medicine, Al-Azhar University and the Research Institute of Ophthalmology Ethical Committee. Also, informed consent was obtained from each participant.

\section{Statistical methodology:}

Analysis was done using SPSS statistical software.

Variables were described by using mean, standard deviation and ranges.

The dependent variables; "Error of Refraction, Best Corrected Visual Acuity, Macular thickness, Choroidal Thickness, FAZ SCP, FAZ DCP", are compared in the two techniques.

Normality assumption is checked for continuous variables "Error of Refraction, Best Corrected Visual Acuity, Macular thickness, Choroidal Thickness, FAZ SCP, FAZ DCP") using Shapiro-Wilk test because of small sample size $(\mathrm{N}=35)$.

Within the Myope population, statistical significance was determined by testing correlations between either continuous variables or continuous and ordinal variables "using spearman rho's correlation coefficient" while between both ordinal variables using "Somer's d test".

Statistical significance was determined using Mann Whitney U test for comparing the not normally distributed continuous variables "error of refraction, Best Corrected Visual Acuity, Macular thickness, Choroidal Thickness, FAZ SCP, FAZ DCP") in the Myope group and Emmetrope group.

\section{RESULTS}

- Number of patients with high Myope $=18$ patients, therefore 36 eye measurements.

- Patients included are high Myope with error of refraction ranging from -6 to $-25 \mathrm{D}$, therefore 1 right eye was excluded due to refraction error of $+1 \mathrm{D}$.

- Number of eye measurements included is 35 eyes.

\section{Descriptive Analysis for Myopic Patients:}

- Mean age of patients $(n=18)$ is $32 \pm 9.7$ (SD) years old, Ranging from 20 to 54 years old.

- Mean error of refraction in eyes $(n=35)$ is $-15.3 \pm 6.6(\mathrm{SD})$, Ranging from -25 to $-6 \mathrm{D}$.

- Mean best corrective of visual acuity of eyes $(n=35)$ is $0.34 \pm 0.2$ (stdev), Ranging from 0.02 to 0.8 .

- Mean Macular thickness of the eyes ( $\mathrm{n}=35$ ) is $155.2 \pm 58.1$ (stdev), Ranging from 76 to 380 .

- Mean Choroidal thickness of the eyes ( $\mathrm{n}=35$ ) is $140.4 \pm 112.9$ (stdev), Ranging from 19 to 454.

- Mean FAZ SCP of the eyes ( $\mathrm{n}=35$ ) is $434 \pm 129.4$ (stdev), Ranging from 250 to 890.

- Mean FAZ DCP of the eyes ( $n=35)$ is $301 \pm 90.4$ (stdev), Ranging from 70 to 479 .

The SCP density, DCP density and CC density are described as the number and percentage of eyes measurements seen in each quadrant as the following:

\begin{tabular}{|c|c|c|}
\hline SCP Density Grade & No of Eyes & Percentage \\
\hline 0 & 6 & $17.14 \%$ \\
\hline 2 & 14 & $40.00 \%$ \\
\hline 3 & 12 & $34.29 \%$ \\
\hline NA & 2 & $5.71 \%$ \\
\hline Grand Total & 1 & $2.86 \%$ \\
\hline DCP Density Grade & $\mathbf{3 5}$ & $\mathbf{1 0 0 . 0 0 \%}$ \\
\hline 0 & No of Eyes & Percentage \\
\hline 1 & 4 & $11.43 \%$ \\
\hline 2 & 11 & $31.43 \%$ \\
\hline 3 & 18 & $51.43 \%$ \\
\hline NA & 1 & $2.86 \%$ \\
\hline Grand Total & 1 & $2.86 \%$ \\
\hline CC Density Grade & $\mathbf{3 5}$ & $\mathbf{1 0 0 . 0 0 \%}$ \\
\hline 0 & No of Eyes & Percentage \\
\hline 1 & 2 & $5.71 \%$ \\
\hline 2 & 3 & $8.57 \%$ \\
\hline
\end{tabular}


Mohamed El-Massry et al.

\begin{tabular}{|c|c|c|}
\hline 3 & 6 & $17.14 \%$ \\
\hline 4 & 1 & $2.86 \%$ \\
\hline NA & 1 & $2.86 \%$ \\
\hline Grand Total & $\mathbf{3 5}$ & $\mathbf{1 0 0 . 0 0 \%}$ \\
\hline
\end{tabular}

\section{Descriptive analysis for Emmetrope patients:}

- Number of Emmetrope patients=20 patients , therefore 40 eye measurements.

- Mean age of patients $(\mathrm{n}=20)$ is $30.6 \pm 8.4$ (stdev) years old, Ranging from 20 to 58 years old.

- Mean best corrective of visual acuity of eyes $(n=40)$ is $0.77 \pm 0.1$ (stdev), Ranging from 0.6 to 0.9 .

- Mean Macular thickness of the eyes ( $n=40)$ is $223.6 \pm 22.3$ (stdev), Ranging from 180 to 262.

- Mean Choroidal thickness of the eyes $(n=40)$ is 330.2 \pm 59.9 (stdev), Ranging from 230 to 456.

- Mean FAZ SCP of the eyes ( $\mathrm{n}=35$ ) is $411 \pm 109.2$ (stdev), Ranging from 140 to 590 .

- Mean FAZ DCP of the eyes ( $\mathrm{n}=35$ ) is $209.3 \pm 57.9$ (stdev), Ranging from 80 to 330 .

Inferential analysis:

In the patients with high Myopia:

- Normality assumption is checked for each continuous variable to carry out correlations using Shapiro-Wilk test (sample size<50).

- From the results of Shapiro-Wilk test all the variables are not normal except for the Best Corrected visual acuity variable.

Tests of Normality

\begin{tabular}{|l|l|l|l|}
\hline \multirow{2}{*}{} & \multicolumn{3}{|c|}{ Shapiro-Wilk } \\
\cline { 2 - 4 } & Statistic & df & Sig. \\
\hline Error of Refraction & .897 & 34 & .004 \\
Best Corrected Visual Acuity & .946 & 34 & .091 \\
Macular thickness & .764 & 34 & .000 \\
Choroidal Thickness & .841 & 34 & .000 \\
FAZ SCP & .891 & 34 & .003 \\
FAZ DCP & .891 & 34 & .003 \\
\hline
\end{tabular}

Correlations results between both continuous variables:

- Correlations are done using The Spearman rank-order correlation coefficient because of not normal and continuous variables.

1- Testing correlation between error of refraction and best corrected visual acuity.

Correlations

\begin{tabular}{|l|l|l|c|c|}
\hline \multicolumn{2}{|c|}{} & $\begin{array}{c}\text { Error of } \\
\text { Refraction }\end{array}$ & $\begin{array}{c}\text { Best Corrected } \\
\text { Visual Acuity }\end{array}$ \\
\hline \multirow{4}{*}{ Spearman's rho } & \multirow{3}{*}{ Error of Refraction } & Correlation Coefficient & 1.000 & $.560^{* *}$ \\
\cline { 3 - 5 } & & Sig. (2-tailed) &. & .000 \\
\cline { 2 - 5 } & $\mathrm{N}$ & 35 & 35 \\
\cline { 2 - 5 } & \multirow{2}{*}{$\begin{array}{l}\text { Best Corrected Visual } \\
\text { Acuity }\end{array}$} & Correlation Coefficient & $.560^{* *}$ & 1.000 \\
\cline { 3 - 5 } & Sig. (2-tailed) & .000 &. \\
\cline { 2 - 5 } & & 35 & 35 \\
\hline
\end{tabular}

* Correlation is significant at the 0.01 level (2-tailed).

Interpretation:

A Spearman's rank-order correlation was run to determine the relationship between Error of refraction and best visual acuity. There was a moderate, positive correlation between Error of refraction and best visual acuity, which was statistically significant $\left(r_{s}=0.56, \mathrm{p}=0.00\right)$.

2- Testing correlation between error of refraction and macular thickness.

Correlations

\begin{tabular}{|c|c|c|c|c|}
\hline & & & Error of Refraction & Macular thickness \\
\hline \multirow{2}{*}{ Spearman's rho } & \multirow{2}{*}{$\begin{array}{ll}\text { Error } & \text { of } \\
\text { Refraction } & \\
\end{array}$} & Correlation Coefficient & 1.000 & -.151 \\
\hline & & Sig. (2-tailed) & & .388 \\
\hline
\end{tabular}




\begin{tabular}{|l|l|l|l|l|}
\hline & $\mathrm{N}$ & 35 & 35 \\
\cline { 2 - 5 } & \multirow{3}{*}{$\begin{array}{l}\text { Macular } \\
\text { thickness }\end{array}$} & Correlation Coefficient & -.151 & 1.000 \\
\cline { 3 - 5 } & Sig. (2-tailed) & .388 &. \\
\cline { 2 - 5 } & $\mathrm{N}$ & 35 & 35 \\
\hline
\end{tabular}

Interpretation:

A Spearman's rank-order correlation was run to determine the relationship between Error of refraction and Macular thickness. There was a very weak, negative correlation between Error of refraction and Macular thickness, which was not statistically significant $\left(r_{s}=-0.151, \mathrm{p}=0.388\right)$.

3- Testing correlation between error of refraction and choroidal thickness.

Correlations

\begin{tabular}{|l|l|l|l|l|}
\hline \multicolumn{2}{|c|}{} & $\begin{array}{l}\text { Error } \\
\text { Refraction }\end{array}$ & $\begin{array}{l}\text { Choroidal } \\
\text { Thickness }\end{array}$ \\
\hline \multirow{4}{*}{ Spearman's rho } & \multirow{3}{*}{ Error of Refraction } & Correlation Coefficient & 1.000 & $.680^{* *}$ \\
\cline { 3 - 5 } & & Sig. (2-tailed) &. & .000 \\
\cline { 2 - 5 } & N & 35 & 35 \\
\cline { 2 - 5 } & \multirow{3}{*}{ Choroidal } & Correlation Coefficient & $.680^{* *}$ & 1.000 \\
\cline { 3 - 5 } & & Sig. (2-tailed) & .000 &. \\
\cline { 3 - 5 } & Thickness & $\mathrm{N}$ & 35 & 35 \\
\hline
\end{tabular}

** Correlation is significant at the 0.01 level (2-tailed). Interpretation:

A Spearman's rank-order correlation was run to determine the relationship between Error of refraction and choroidal thickness. There was a strong, positive correlation between Error of refraction and choroidal thickness, which was statistically significant $\left(r_{s}=0.68, \mathrm{p}=0.00\right)$.

4- Testing correlation between error of refraction and FAZ SCP.

Correlations

\begin{tabular}{|c|c|c|c|c|}
\hline & & & Error of Refraction & FAZ SCP \\
\hline \multirow{6}{*}{ Spearman's rho } & \multirow{3}{*}{$\begin{array}{l}\text { Error } \\
\text { Refraction }\end{array}$} & Correlation Coefficient & 1.000 & .187 \\
\hline & & Sig. (2-tailed) & 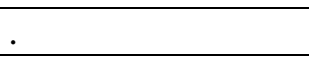 & .288 \\
\hline & & $\mathrm{N}$ & 35 & 34 \\
\hline & \multirow{3}{*}{ FAZ SCP } & Correlation Coefficient & .187 & 1.000 \\
\hline & & Sig. (2-tailed) & .288 & . \\
\hline & & $\mathrm{N}$ & 34 & 34 \\
\hline
\end{tabular}

Interpretation:

A Spearman's rank-order correlation was run to determine the relationship between Error of refraction and FAZ SCP. There was a very weak, positive correlation between Error of refraction and FAZ SCP, which was not statistically significant $\left(r_{s}=0.187, \mathrm{p}=0.288\right)$.

5- Testing correlation between error of refraction and FAZ DCP.

\section{Correlations}

\begin{tabular}{|c|c|c|c|c|}
\hline & & & $\begin{array}{l}\text { Error of } \\
\text { Refraction }\end{array}$ & $\begin{array}{l}\text { FAZ } \\
\text { DCP(n) }\end{array}$ \\
\hline \multirow{6}{*}{ Spearman's rho } & \multirow{3}{*}{$\begin{array}{l}\text { Error of } \\
\text { Refraction }\end{array}$} & Correlation Coefficient & 1.000 & -.044 \\
\hline & & Sig. (2-tailed) & . & .804 \\
\hline & & $\mathrm{N}$ & 35 & 35 \\
\hline & \multirow{3}{*}{ FAZ DCP(n) } & Correlation Coefficient & -.044 & 1.000 \\
\hline & & Sig. (2-tailed) & .804 & . \\
\hline & & $\mathrm{N}$ & 35 & 40 \\
\hline
\end{tabular}


Interpretation:

A Spearman's rank-order correlation was run to determine the relationship between Error of refraction and FAZ DCP. There was a very weak, negative correlation between Error of refraction and FAZ DCP, which was not statistically significant $\left(r_{s}=-0.044, \mathrm{p}=0.804\right)$

6- Testing correlation between error of refraction and age

\section{Correlations}

\begin{tabular}{|l|l|l|l|l|}
\hline \multicolumn{1}{|c|}{} & \multirow{3}{*}{$\begin{array}{l}\text { Error } \\
\text { Refraction }\end{array}$} & $\begin{array}{l}\text { Correlation } \\
\text { Coefficient }\end{array}$ & Error of Refraction & Age per eye \\
\hline & Sig. (2-tailed) & 1.000 & -.111 \\
\cline { 2 - 5 } Spearman's rho & $\mathrm{N}$ & 35 & .527 \\
\cline { 2 - 5 } & \multirow{3}{*}{ Age per eye } & $\begin{array}{l}\text { Correlation } \\
\text { Coefficient }\end{array}$ & -.111 & 35 \\
\cline { 3 - 5 } & Sig. (2-tailed) & .527 & 1.000 \\
\cline { 3 - 5 } & $\mathrm{N}$ & 35 & 35 \\
\hline
\end{tabular}

Interpretation:

A Spearman's rank-order correlation was run to determine the relationship between Error of refraction and age. There was a very weak, negative correlation between Error of refraction and age, which was not statistically significant $\left(r_{s}=-0.111, \mathrm{p}=0.527\right)$

Correlations results between error of refraction continuous variable and SCP density, DCP density, CC density ordinal variables:

- Correlations are done using Spearman's rank-order correlation coefficient.

- The SCP, DCP \& CC densities are scaled on a scale from 0-4, 0 the most abnormal to 4 the most normal.

1- Correlation between Error of refraction and SCP density:

Correlations

\begin{tabular}{|l|l|l|l|l|}
\hline \multicolumn{3}{|c|}{} & Error of Refraction & SCP density 2 \\
\hline \multirow{4}{*}{ Spearman's rho } & \multirow{3}{*}{ Error of Refraction } & Correlation Coefficient & 1.000 & $.663^{* *}$ \\
\cline { 3 - 5 } & & Sig. (2-tailed) &. & .000 \\
\cline { 2 - 5 } & $\mathrm{N}$ & 35 & 34 \\
\cline { 2 - 5 } & \multirow{3}{*}{ SCP density 2 } & Correlation Coefficient & $.663^{* *}$ & 1.000 \\
\cline { 3 - 5 } & Sig. (2-tailed) & .000 &. \\
\cline { 3 - 5 } & $\mathrm{N}$ & 34 & 34 \\
\hline
\end{tabular}

**. Correlation is significant at the 0.01 level (2-tailed).

Interpretation:

A Spearman's rank-order correlation was run to determine the relationship between Error of refraction and SCP density. There was a strong, positive correlation between Error of refraction and SCP density, which was statistically significant $\left(r_{s}=0.663, \mathrm{p}=0.00\right)$ (Therefore as the error of refraction moves toward -6 , the scp density moves to resemble the normal more ( 4 on the scale) and vice versa).

2- Correlation between Error of refraction and DCP density:

Correlations

\begin{tabular}{|l|l|l|l|l|}
\hline \multicolumn{3}{|c|}{} & Error of Refraction & DCP density 2 \\
\hline \multirow{3}{*}{ Spearman's rho } & \multirow{3}{*}{ Error of Refraction } & Correlation Coefficient & 1.000 & $.386^{*}$ \\
\cline { 3 - 5 } & & Sig. (2-tailed) &. & .024 \\
\cline { 2 - 5 } & $\mathrm{N}$ & 35 & 34 \\
\cline { 2 - 5 } & \multirow{2}{*}{ DCP density 2 } & Correlation Coefficient & $.386^{*}$ & 1.000 \\
\cline { 3 - 5 } & Sig. (2-tailed) & .024 &. \\
\hline
\end{tabular}




\begin{tabular}{|l|l|l|l|l|}
\hline & & $\mathrm{N}$ & 34 & 34 \\
\hline
\end{tabular}

*. Correlation is significant at the 0.05 level (2-tailed).

\section{Interpretation:}

A Spearman's rank-order correlation was run to determine the relationship between Error of refraction and DCP density. There was a weak, positive correlation between Error of refraction and DCP density, which was statistically significant $\left(r_{s}=0.386, \mathrm{p}=0.024\right)$

3- Correlation between Error of refraction and CC density:

\begin{tabular}{|c|c|c|c|c|}
\hline \multicolumn{5}{|l|}{ Correlations } \\
\hline & & & Error of Refraction & CC density 2 \\
\hline \multirow{6}{*}{ Spearman's rho } & \multirow{3}{*}{ Error of Refraction } & Correlation Coefficient & 1.000 & $.487^{* *}$ \\
\hline & & Sig. (2-tailed) & . & .003 \\
\hline & & $\mathrm{N}$ & 35 & 34 \\
\hline & \multirow{3}{*}{ CC density 2} & Correlation Coefficient & $.487^{* *}$ & 1.000 \\
\hline & & Sig. (2-tailed) & .003 & . \\
\hline & & $\mathrm{N}$ & 34 & 34 \\
\hline
\end{tabular}

Interpretation:

A Spearman's rank-order correlation was run to determine the relationship between Error of refraction and $\mathrm{CC}$ density. There was a moderate, positive correlation between Error of refraction and CC density, which was statistically significant $\left(r_{s}=0.487, \mathrm{p}=0.003\right)$

- The error of refraction is divided to three categories: (1) less than -15 , (2) less than -10 to -15 , (3) -6 to -10 .

- Correlations between the ordinal variable (error of refraction ranges) and the continuous variables (Best visual acuity, macular thickness, choroidal thickness and age) are done using Spearman's rho correlation coefficient.

1- Correlation between error of refraction ranges and best visual acuity:

\section{Correlations}

\begin{tabular}{|l|l|l|l|l|}
\hline \multicolumn{2}{|c|}{} & $\begin{array}{l}\text { error of refraction } \\
\text { ranges }\end{array}$ & $\begin{array}{l}\text { Best Corrected } \\
\text { Visual Acuity }\end{array}$ \\
\hline \multirow{4}{*}{$\begin{array}{l}\text { Spearman's rho } \\
\text { error of refraction ranges }\end{array}$} & Correlation Coefficient & 1.000 & $.559^{* *}$ \\
\cline { 2 - 5 } & Sig. (2-tailed) &. & .000 \\
\cline { 2 - 5 } & $\mathrm{N}$ & 35 & 35 \\
\cline { 2 - 5 } & $\begin{array}{l}\text { Best Corrected Visual } \\
\text { Acuity }\end{array}$ & Correlation Coefficient & $.559^{* *}$ & 1.000 \\
\cline { 3 - 5 } & Sig. (2-tailed) & .000 &. \\
\cline { 2 - 4 } & $\mathrm{N}$ & 35 & 35 \\
\hline
\end{tabular}

Interpretation:

A Spearman's rank-order correlation was run to determine the relationship between Error of refraction ranges and Best visual acuity. There was a moderate, positive correlation between Error of refraction ranges and best visual acuity, which was statistically significant $\left(r_{s}=0.559, \mathrm{p}=0.000\right)$

2- Correlation between error of refraction ranges and macular thickness:

\section{Correlations}

\begin{tabular}{|l|l|l|l|l|}
\hline \multicolumn{2}{|l|}{} & $\begin{array}{l}\text { error of refraction } \\
\text { ranges }\end{array}$ & $\begin{array}{l}\text { Macular } \\
\text { thickness }\end{array}$ \\
\hline \multirow{2}{*}{ Spearman's rho } & \multirow{2}{*}{ error of refraction ranges } & Correlation Coefficient & 1.000 & -.196 \\
\cline { 3 - 5 } & Sig. (2-tailed) &. & .259 \\
\hline
\end{tabular}


Mohamed El-Massry et al.

\begin{tabular}{|l|l|l|l|l|}
\hline \multirow{3}{*}{ Macular thickness } & $\mathrm{N}$ & 35 & 35 \\
\cline { 2 - 5 } & & Correlation Coefficient & -.196 & 1.000 \\
\cline { 2 - 5 } & Sig. (2-tailed) & .259 &. \\
\cline { 2 - 4 } & $\mathrm{N}$ & 35 & 35 \\
\hline
\end{tabular}

Interpretation:

A Spearman's rank-order correlation was run to determine the relationship between Error of refraction ranges and macular thickness. There was a very weak, positive correlation between Error of refraction ranges and macular thickness, which was not statistically significant $\left(r_{s}=-0.196, \mathrm{p}=0.259\right)$.

3- Correlation between error of refraction ranges and choroidal thickness:

\section{Correlations}

\begin{tabular}{|l|l|l|l|l|}
\hline \multicolumn{2}{|c|}{} & $\begin{array}{l}\text { error of refraction } \\
\text { ranges }\end{array}$ & $\begin{array}{l}\text { Choroidal } \\
\text { Thickness }\end{array}$ \\
\hline \multirow{4}{*}{$\begin{array}{l}\text { error of refraction } \\
\text { Sanges }\end{array}$} & Correlation Coefficient & 1.000 & $.637^{* *}$ \\
\cline { 3 - 5 } & Sig. (2-tailed) &. & .000 \\
\cline { 2 - 5 } & $\mathrm{N}$ & 35 & 35 \\
\cline { 2 - 5 } & \multirow{3}{*}{ Choroidal Thickness } & Correlation Coefficient & $.637^{* *}$ & 1.000 \\
\cline { 3 - 5 } & Sig. (2-tailed) & .000 &. \\
\cline { 2 - 5 } & $\mathrm{N}$ & 35 & 35 \\
\hline
\end{tabular}

Interpretation:

A Spearman's rank-order correlation was run to determine the relationship between Error of refraction ranges and choroidal thickness. There was a strong, positive correlation between Error of refraction ranges and choroidal thickness, which was statistically significant $\left(r_{s}=0.637, \mathrm{p}=0.000\right)$

4- Correlation between error of refraction ranges and age:

Correlations

\begin{tabular}{|c|c|c|c|c|}
\hline & & & $\begin{array}{l}\text { error of refraction } \\
\text { ranges }\end{array}$ & Age per eye \\
\hline \multirow{6}{*}{ Spearman's rho } & \multirow{3}{*}{$\begin{array}{l}\text { error of refraction } \\
\text { ranges }\end{array}$} & Correlation Coefficient & 1.000 & -.058 \\
\hline & & Sig. (2-tailed) & & .742 \\
\hline & & $\mathrm{N}$ & 35 & 35 \\
\hline & \multirow{3}{*}{ Age per eye } & Correlation Coefficient & -.058 & 1.000 \\
\hline & & Sig. (2-tailed) & .742 & \\
\hline & & $\mathrm{N}$ & 35 & 35 \\
\hline
\end{tabular}

Interpretation:

A Spearman's rank-order correlation was run to determine the relationship between Error of refraction ranges and age. There was a very weak, negative correlation between Error of refraction ranges and ages, which was not statistically significant $\left(r_{s}=-0.058, \mathrm{p}=0.742\right)$

These categories are correlated with the rest of the variables using Somers'd test because both variables are ordinal.

1- Correlation between error of refraction ranges and SCPdensity:

Directional Measures

\begin{tabular}{|l|l|l|l|l|l|l|}
\hline \multicolumn{2}{|l|}{} & \multicolumn{1}{|c|}{ Value } & $\begin{array}{c}\text { Asymp. Std. } \\
\text { Error }^{\mathrm{a}}\end{array}$ & \multicolumn{1}{|c|}{$\begin{array}{l}\text { Approx. } \mathrm{T}^{\mathrm{b}} \\
\text { Approx. } \\
\text { Sig. }\end{array}$} \\
\hline $\begin{array}{l}\text { Ordinal by } \\
\text { Ordinal }\end{array}$ & Somers'd d & Symmetric & .601 & .104 & 5.388 & .001 \\
\cline { 2 - 7 } & $\begin{array}{l}\text { error of refraction ranges } \\
\text { Dependent }\end{array}$ & .593 & .096 & 5.388 & .001 \\
\hline
\end{tabular}




\begin{tabular}{|c|c|c|c|}
\hline & SCP density 2 Dependent & 610 & 11 \\
\hline
\end{tabular}

Interpretation:

Somers'd was run to determine the association between Error of refraction and SCP density. There was a strong, positive correlation between Error of refraction and SCP density, which was statistically significant $(\mathrm{d}=0.610, \mathrm{p}=0.000)$.

2- Correlation between error of refraction ranges and DCP density:

\section{Directional Measures}

\begin{tabular}{|l|l|l|l|l|l|l|}
\hline \multicolumn{2}{|c|}{} & Value & $\begin{array}{c}\text { Asymp. } \\
\text { Std. Error }\end{array}$ & $\begin{array}{c}\text { Approx. } \\
\mathrm{T}^{\mathrm{b}}\end{array}$ & Approx. Sig. \\
\hline \multirow{3}{*}{$\begin{array}{l}\text { Ordinal } \\
\text { by } \\
\text { Ordinal }\end{array}$} & Somers' d & .392 & .134 & 2.766 & .006 \\
\cline { 2 - 6 } & $\begin{array}{l}\text { Symmetric } \\
\begin{array}{l}\text { error of refraction } \\
\text { ranges Dependent }\end{array}\end{array}$ & .409 & .133 & 2.766 & .006 \\
\cline { 2 - 6 } & $\begin{array}{l}\text { DCP density } \\
\text { Dependent }\end{array}$ & .377 & .137 & 2.766 & .006 \\
\hline
\end{tabular}

Interpretation:

Somers'd was run to determine the association between Error of refraction and DCP density. There was a weak, positive correlation between Error of refraction and DCP density, which was statistically significant $(\mathrm{d}=0.377, \mathrm{p}=0.006)$.

3- Correlation between error of refraction ranges and CC density:

Directional Measures

\begin{tabular}{|c|c|c|c|c|c|c|}
\hline & Value & $\begin{array}{l}\text { Asymp. Std. } \\
\text { Error }^{\mathrm{a}}\end{array}$ & Approx. $\mathrm{T}^{\mathrm{b}}$ & Approx. Sig. \\
\hline \multirow{3}{*}{$\begin{array}{l}\text { Ordinal by } \\
\text { Ordinal }\end{array}$} & \multirow{3}{*}{ Somers' d } & Symmetric & .468 & .114 & 3.470 & .001 \\
\hline & & $\begin{array}{l}\text { error of refraction } \\
\text { ranges Dependent }\end{array}$ & .518 & .114 & 3.470 & .001 \\
\hline & & $\begin{array}{ll}\text { CC density } & 2 \\
\text { Dependent } & \end{array}$ & .427 & .125 & 3.470 & .001 \\
\hline
\end{tabular}

Interpretation:

Somers'd was run to determine the association between Error of refraction and CC density. There was a moderate, positive correlation between Error of refraction and CC density, which was statistically significant $(\mathrm{d}=0.427, \mathrm{p}=0.001)$.

\section{Correlating the patients with High $\operatorname{Myope}(n=35)$ with the normal patients $(n=40)$.}

- Patients with high Myope are coded (1), Normal patients are coded (2).

Normality assumption is checked for each group of the independent variable (Myope patients or normal patients) using Shapiro-Wilk test (sample size<50). 
Tests of Normality

\begin{tabular}{|l|c|c|c|c|}
\hline \multirow{2}{*}{ Patients } & \multicolumn{3}{|c|}{ Shapiro-Wilk } \\
\cline { 3 - 5 } & & Statistic & df & Sig. \\
\hline \multirow{2}{*}{ Best corrective visual acuity } & 1 & .946 & 34 & .091 \\
\cline { 2 - 5 } & 2 & .846 & 40 & .001 \\
\hline \multirow{2}{*}{ Macular thickness } & 1 & .764 & 34 & .001 \\
\cline { 2 - 5 } & 2 & .961 & 40 & .175 \\
\hline \multirow{2}{*}{ Choroidal thickness } & 1 & .841 & 34 & .001 \\
\cline { 2 - 5 } & 2 & .959 & 40 & .157 \\
\hline \multirow{2}{*}{ FAZ SCP } & 1 & .895 & 34 & .003 \\
\cline { 2 - 5 } & 2 & .891 & 40 & .001 \\
\hline \multirow{2}{*}{ FAZ DCP } & 1 & .947 & 34 & .102 \\
\cline { 2 - 5 } & 2 & .968 & 40 & .313 \\
\hline
\end{tabular}

- From the results of Shapiro-Wilk test all the variables are not normal for each group of the independent variable.

- Therefore, Mann-Whitney U test is used to correlate the continuous variables in both groups.

\section{Ranks}

\begin{tabular}{|c|c|c|c|c|}
\hline & Patients & $\mathrm{N}$ & Mean Rank & Sum of Ranks \\
\hline \multirow{3}{*}{$\begin{array}{l}\text { Best corrective visual } \\
\text { acuity }\end{array}$} & 1 & 35 & 19.57 & 685.00 \\
\hline & 2 & 40 & 54.13 & 2165.00 \\
\hline & Total & 75 & & \\
\hline \multirow{3}{*}{ Macular thickness } & 1 & 35 & 21.57 & 755.00 \\
\hline & 2 & 40 & 52.38 & 2095.00 \\
\hline & Total & 75 & & \\
\hline \multirow{3}{*}{ Choroidal thickness } & 1 & 35 & 21.44 & 750.50 \\
\hline & 2 & 40 & 52.49 & 2099.50 \\
\hline & Total & 75 & & \\
\hline \multirow{3}{*}{ FAZ SCP } & 1 & 34 & 37.21 & 1265.00 \\
\hline & 2 & 40 & 37.75 & 1510.00 \\
\hline & Total & 74 & & \\
\hline \multirow{3}{*}{ FAZ DCP } & 1 & 34 & 50.46 & 1715.50 \\
\hline & 2 & 40 & 26.49 & 1059.50 \\
\hline & Total & 74 & & \\
\hline
\end{tabular}

The table above indicates that the normal patients group has a higher mean rank of the Best corrective visual acuity, Macular thickness, choroidal thickness and FAZ SCP but the Myope patients have higher mean rank of the FAZ DCP.

\section{Test Statistics ${ }^{\mathrm{a}}$}

\begin{tabular}{|l|c|c|c|c|c|}
\hline & $\begin{array}{c}\text { Best corrective } \\
\text { visual acuity }\end{array}$ & $\begin{array}{c}\text { Macular } \\
\text { thickness }\end{array}$ & $\begin{array}{c}\text { Choroidal } \\
\text { thickness }\end{array}$ & FAZ SCP & FAZ DCP \\
\hline Mann-Whitney U & 55.000 & 125.000 & 120.500 & 670.000 & 239.500 \\
\hline Wilcoxon W & 685.000 & 755.000 & 750.500 & 1265.000 & 1059.500 \\
\hline Z & -6.908 & -6.112 & -6.156 & -.109 & -4.780 \\
\hline Asymp. Sig. (2-tailed) & .000 & .000 & .000 & .914 & .001 \\
\hline a. Grouping Variable: patients
\end{tabular}

From this data, it can be concluded that:

- The Best corrective visual acuity in the normal group was statistically significantly higher than the Myope group $(U=55, p=0.000)$.
- The Macular thickness in the normal group was statistically significantly higher than the Myope group $(U=125, p=0.000)$. 
- The choroidal thickness in the normal group was statistically significantly higher than the Myope group $(U=120.5, p=0.000)$.

- The FAZ SCP in the normal group was not statistically significantly higher than the Myope group $(U=670, p=0.914)$.

- The FAZ DCP in the Myope group was statistically significantly higher than the normal group $(U=$ $239, p=0.000$ ).

\section{DISCUSSION}

This prospective study is concerned with observing retinal and choroidal changes in high myopia. Using EDI-OCT, macular thickness and choroidal thickness were assessed, whereas the SCP, DCP and CC density were assessed using OCT angio. 40 normal eyes and 35 high myopic eyes were examined and the findings were statistically compared to the error of refraction of each eye.

One of the parameters assessed in our study is the subfoveal choroidal thickness SFCT. This was measured using EDI-OCT measuring from the outer layer of the RPE to the choroido scleral junction. Results showed that the mean Choroidal thickness of the high myopic eyes $(n=35)$ is $140.4 \mathrm{um} \pm 112.9 \mathrm{um}$ (stdev), Ranging from 19um to 454um. While in emmetrope eyes Mean Choroidal thickness of the eyes $(\mathrm{n}=40)$ is $330.2 \mathrm{um} \pm 59.9 \mathrm{um}$ (stdev), ranging from 230 um to $456 u m$. There was a strong, positive correlation between Error of refraction and choroidal thickness, denoting that the choroidal thickness decreases as the degree of error of refraction increases. The choroidal thickness in the normal group was statistically significantly higher than the Myope group $(U=120.5, p=0.000)$.

Teberik et $\boldsymbol{a l} .{ }^{(12)}$ concluded that the subfoveal CT was significantly lower in the high myopia group (mean, $218 \mathrm{um}$ ) than the control group (mean, 331um) It had 65 individuals (30 patients with high myopia, 35 healthy subjects).

In highly myopic eyes, excessive axial elongation of the eyeballs can cause biomechanical stretching and thinning of the choroid, retina, and sclera $^{(\mathbf{1 3 )}}$. Ikuno et $\boldsymbol{a l}^{\left({ }^{(14)}\right.}$ showed significant associations between choroidal thickness, error of refraction, CT, and posterior staphyloma height but not AL. The tessellated group in a study by Wang et al. showed no correlation between AL/error of refraction and $\mathrm{CT}$, despite the fact that the diffuse chorioretinal atrophy group (with higher AL and error of refraction) showed strong correlations with both variables. Takahashi et $\boldsymbol{a l}^{(\mathbf{1 5 )}}$ observed a correlation between $\mathrm{CT}$ and $\mathrm{AL}$, and Chen et $\boldsymbol{a l} .{ }^{(16)}$ found one between CT and error of refraction.
Guptaet al. ${ }^{(17)}$ stated that the CT at the subfovea was significantly thinner (mean $225 \mathrm{um}$ ) for high myopes compared to emmetropes (375.15 um) based on a case-control study of 648 young, male subjects, including 520 high myopes and 128 emmetropes.

Ignacio et $\mathbf{a l} .^{(18)}$ concluded that mean macular choroidal thickness was 115.5 um (range, 6.9-436.0) after examination of a total of 120 eyes of 83 patients with high myopia (error of refraction $\geq-6$ diopters or axial length $\geq 26 \mathrm{~mm}$ ) and 96 eyes of 62 healthy patients. They reported that the relationship between age and $\mathrm{CT}$ in patients with high myopia was similar to that of a control group after they accounted for the effects of AL. They also suggested that AL has the strongest effect on CT in highly myopic eyes. Also they found out that BCVA $(\log M A R)$ was significantly correlated with thinner SFCT.

El-Shazly et $a .^{(19)}$ found Choroidal thickness was significantly lower in myopic eyes compared to controls. Regardless of the degree of myopia, nasal regions showed the lowest CT with decremental pattern with advance of myopia (low myopia $279.00 \pm 24.50 \mu \mathrm{m}$, moderate myopia $269.58 \pm 20.69 \mu \mathrm{m}$, high myopia $189.58 \pm 25.95 \mu \mathrm{m}$, advanced myopia $96.75 \pm 24.83 \mu \mathrm{m}) .240$ patients with myopia and 60 emmetropes as controls.

Another assessed parameter is the central macular thickness CMT. Using also EDI-OCT thickness was measured first manual from the inner layer of the ILM to the inner layer of the RPE then reassured by the value obtained by the macular thickness map. Mean Macular thickness of the myopic eyes $(\mathrm{n}=35)$ is $155.2 \mathrm{um} \pm 58.1 \mathrm{um}(\mathrm{stdev})$, Ranging from $76 \mathrm{um}$ to $380 \mathrm{um}$. Mean Macular thickness of the emmetrope eyes $(n=40)$ is $223.6 \mathrm{um}$ \pm 22.3 um (stdev), Ranging from 180 um to $262 \mathrm{um}$. There was a very weak, negative correlation between error of refraction and macular thickness, denoting that increase in the error of refraction is not accompanied by decrease in macular thickness. However the Macular thickness in the normal group was statistically significantly higher than the Myope group. Consistent with our results, Abdulwaris et al. ${ }^{(20)}$, found out that the overall mean macular thickness in the myopic groups and control were $262.98 \mathrm{um}$ and $290.92 \mathrm{um}$ respectively. The total macular thickness was less in myopic eyes as compared to emmetropic eyes. Minghui et al. (21) also showed that error of refraction was negatively correlated with central macular thickness, which is also approved by Barrio-Barrio et al. ${ }^{(22)}$ and AlHaddad et al. $^{(23)}$.

Using OCT-angio, assessment of the foveal avascular zone FAZ was performed, in both SCP \& 
DCP. Results showed that in myopic eyes the mean FAZ in SCP is $434 \mathrm{um}^{2} \pm 129.4 \mathrm{um}^{2}$ (stdev), Ranging from $250 \mathrm{um}^{2}$ to $890 \mathrm{um}^{2}$. While the mean FAZ in $\mathrm{DCP}$ is $301 \mathrm{um}^{2} \pm 90.4 \mathrm{um}^{2}$ (stdev), Ranging from $70 \mathrm{um}^{2}$ to $479 \mathrm{um}^{2}$. On the other hand, in emmetrope eyes the mean FAZ area in SCP is $411 \mathrm{um}^{2} \pm$ $109.2 \mathrm{um}^{2}$ (stdev), Ranging from $140 \mathrm{um}^{2}$ to $590 \mathrm{um}^{2}$. While the mean FAZ in DCP is $209.3 \mathrm{um}^{2} \pm 57.9 \mathrm{um}^{2}$ (stdev), Ranging from $80 \mathrm{um}^{2}$ to $330 \mathrm{um}^{2}$. To date, there are only a few articles describing FAZ area measured with OCT-angio ${ }^{(24)}$, with the mean area of the superficial FAZ ranging from 250 to $304 \mathrm{um}^{2}$, while the deep FAZ ranges from 340 to $495 \mathrm{um}^{2(25)}$. In our study, despite following the exact same method used in the above mentioned studies, our results showed that the FAZ in SCP is larger in both myope and emmmetrope eyes than the DCP FAZ. This is likely due to difference in the device used as in these studies AngioVue OCT-A system (Optovue, Fremont, CA, USA) was used while in our study Heidelberg engineering, OCT spectralis, Germany device is used. The official Heidelberg website displays an image of the FAZ area in SCP \& DCP of a normal eye showing clearly that the FAZ in SCP larger than the FAZ in DCP.It is also important to note that FAZ size among normal individuals appears to vary greatly at both the superficial and deep levels ${ }^{(24)}$.

In this study, results showed negative correlation between error of refraction and the FAZ size. This is consistent with Colin et $\boldsymbol{a l} .{ }^{(26)}$ In an earlier study on FAZ size using FA, Bresnick $\boldsymbol{e t}$ $\boldsymbol{a l}{ }^{(27)}$ reported that there is no relationship between error of refraction and the circumference or diameter of the FAZ. Similarly, Chui et al. $^{(28)}$ and Dubis et $\boldsymbol{a l}{ }^{(\mathbf{2 9})}$ have reported no significant association between FAZ size and axial length.

Also this study showed that the FAZ in SCP in emmetrope eyes was slightly larger than in myopic eyes while FAZ IN DCP was larger in myope. The latter finding is consistent with MI SUN SUNG et al study which concluded that superficial and deep FAZ were larger compared to emmetropic eyes ${ }^{(30)}$

Previous studies perfomed to assess the vascular density in the SCP, DCP and CC used OCTA with RTVue XR OCT Avanti system (Optovue Inc., Software Version 2015.1.0, Fremont, California, USA). ${ }^{(31)}$ While one study used The Zeiss HD-OCT with AngioplexTM OCTA device (Carl Zeiss Meditec, Dublin, CA). ${ }^{(32)}$

The device used in this study is the Heidelberg engineering, OCT spectralis, Germany didn't have the option to provide a quantitative value of the vascular density like the previously mentioned studies. Moreover there isn't any published study using the abovementioned device in assessment of the vascular plexus. To overcome this challenge, enface OCTA scans acquired over a $3 \mathrm{~mm} \times 3 \mathrm{~mm}$ area over the macula to obtain images of the SCP DCP and CC like the previous studies. Then for each eye of the control group and the high myopia group, the 3 clearly detailed images were assessed morphologically. In myopic eyes the images were assessed compared to the images of the control eyes. The image is divided into 4 quadrants and the density of the vascular plexus is assessed compared to the normal control group, then a grade from 0 to 4 is given with 4 is the best unaffected density (which was the case with all of the 40 emmetropic eyes) and 0 is the worst. If one quadrant is affected morphologically a grade of 3 is given, if 2 quadrants are affected a grade of 2 is given and so on with a grade of 0 if all 4 quadrants are affected. The extent of vascular density in all three layers SCP, DCP $\& C C$ was clearly visible in the acquired images ensuring an accurate grading of all myopic eyes investigated and precisely compared to the control group eyes density. This method was used by Coscas et al. $^{(33)}$ in their study where they evaluated SCP and DCP in retinal vein occlusion morphologically using OCTA.

Our results, consistent with previous study ${ }^{(31)}$, showed a positive correlation between degree of myopia and vascular density in all the 3 layers. All 40 emmetropic eyes, have normal morphological appearance of the vascular plexus density. On the other hand the 35 myopic eyes showed clearly a decrease in vascular density which increased as the degree of myopia increased. Regarding the SCP density, the majority of the eyes (40\%) were of grade 1 meaning that 3 quadrants of the image were clearly morphologically affected compared to a normal eye, while $(34 \%)$ of the eyes were of grade 2 and $(17.14 \%)$ grade 0 and $(5.71 \%)$ of grade 3 . Regarding the DCP density the majority of the eyes $(51.43 \%)$ were of grade $2,(31.43 \%)$ grade $1,(11.43 \%)$ grade 0 and $(2.86 \%)$ grade 3 . Regarding $\mathrm{CC}$ density, the majority of patients $(62.86 \%)$ were of grade 2 while $(17.14 \%)$ grade $3,(8.57 \%)$ grade 1 and $(5.71 \%)$ grade 0 . There was a positive correlation between error of refraction and density in SCP, DCP and CC. This denotes that as the error of refraction increases the vascular density in these 3 layers significantly decreases.

Al-Sheikh et $\boldsymbol{a l}^{\left({ }^{(31)}\right.}$ assessed fifty eyes with myopia and 34 age-matched healthy eyes were included in this study. The vessel density and the vessel branching complexity using fractal dimension 
of the retinal capillary microvasculature were significantly lower in myopic eyes.

The mechanism of decreased retinal and choroidal vascular plexus density and perfusion in high myopic eyes is still unclear. Studies demonstrated that retinal and choroidal thickness decreased in high myopia. ${ }^{(30)}$. Sun Sung et al..$^{(30)}$ highlighted how longer eyes had decreased retinal function and oxygen consumption. Moreover, the structural elongation of the eyeball mechanically stretches the retinal tissue, resulting in the decrease of the retinal microvascular density. It was commonly thought that in pathologically myopic eyes excessive elongation of the eyeball could cause thinning of the retina and choroid, and the thinning of these tissues might decrease the oxygen demand and consequently decrease the blood circulation.

Also it is possible that, with the progress of myopia, the thinned retina due to the elongated axial length can cause degeneration of retinal vascular endothelial cells and retinal pigment epithelium (RPE) cells, resulting in consequential decreases in VEGF production and microvessel density. VEGF produced by vascular endothelial cells and retinal pigment epithelium (RPE) cells, may play a key role in the development of retinal vasculature by stimulating the vessel growth ${ }^{(34)}$. The decreased aqueous VEGF level found in high myopia may also contribute to the possible loss of capillary network ${ }^{(35)}$.

\section{CONCLUSION}

After assessing the images obtained from the 35 high myope eyes and 40 emmetrope eyes using EDI-OCTA, results showed that the choroidal thickness decreases significantly with increase in the error of refraction. Most likely this happens due to axial elongation of high myopic eyes leading to stretch and thinning of the retina. Also, macular thickness decreased in the myopic eyes, which is most likely due to the same theory but there was no relation with error of refraction.

This study is one of the first few studies that assessed FAZ area in high myopia using OCTA. No positive correlation was found between error of refraction and FAZ area but the FAZ area in DCP was larger in myopic eyes compared to the normal group. This might be due to stretching and elongation of the retina and decrease in the vascular density. The vascular density in SCP, DCP \& CC was clearly assessed using OCTA , showing a significant decrease in high myopia and positive correlation with error of refraction.

The OCTA is certainly able to take over other invasive imaging techniques and new researches using this device might be able to study different pathologies affecting the posterior segment.

\section{REFERENCES}

1. Novotny HR, Alvis DL (1961): A method of photographing fluorescence in circulating blood in the human retina. Circulation, 24:82-6.

2. Novotny HR, Alvis DL (1960): A method of photographing fluorescence in circulating blood of the human eye. Tech Doc Rep SAMTDR USAF Sch Aerosp Med., 82:1-4.

3. Kogure K, Choromokos E (1969): Infrared absorption angiography. J Appl Physiol., 26(1):154-7.

4. Yannuzzi LA, Slakter JS, Sorenson JA, Guyer DR, Orlock DA(1992): Digital indocyanine green videoangiography and choroidal neovascularization. Retina,12(3):191-223.

5. Mendis KR, Balaratnasingam C, Yu P et al. (2010): Correlation of histologic and clinical images to determine the diagnostic value of fluorescein angiography for studying retinal capillary detail. Invest Ophthalmol Vis Sci., 51(11): 5864-5869.

6. Staurenghi G, Bottoni F, Giani A (2013): Clinical Applications of Diagnostic Indocyanine Green Angiography. In: Ryan SJ, Sadda SR, Hinton DR (editors), Retina. London: Elsevier Saunders.

7. Bailey ST, Wilson DJ, Tan O et al. (2014): Quantitative optical coherence tomography angiography of choroidal neovascularization in agerelated macular degeneration. Ophthalmology, 21(7):1435-44.

8. de Carlo TE, Bonini Filho MA, Chin AT et al. (2015): Spectral-domain optical coherence tomography angiography of choroidal neovascularization. Ophthalmology, 122(6):1228-38.

9. Weinhaus RS, Burke JM, Delori FC et al. (1995): Comparison of fluorescein angiography with microvascular anatomy of macaque retinas. Exp Eye Res., 61:1-16.

10. Yu PK, Balaratnasingam C, Cringle SJ et al. (2010): Microstructure and network organization of the microvasculature in the human macula. Invest Ophthalmol Vis Sci., 51:6735-6743.

11. Chan G, Balaratnasingam C, Yu PK et al. (2012): Quantitative mor- phometry of perifoveal capillary networks in the human retina. Invest Ophthalmol Vis Sci., 53:5502-5514.

12. Teberik K, Kaya M (2017): Retinal and Choroidal Thickness in Patients with High Myopia without Maculopathy. Pak J Med Sci., 33(6):1438-1443.

13. Wu PC, Chen YJ, Chen CH et al. (2008): Assessment of macular retinal thickness and volume in normal eyes and highly myopic eyes with thirdgeneration optical coherence tomography. Eye (Lond), 22(4):551-555.

14. Ikuno Y, Tano Y (2009): Retinal and choroidal biometry in highly myopic eyes with spectral-domain optical coherence tomography. Invest Ophthalmol Vis Sci., 50(8):3876-3880. 
15. Takahashi A, Ito Y, Iguchi Y et al. (2012): Axial length increases and related changes in highly myopic normal eyes with myopic complications in fellow eyes. Retina, 32(1):127-133.

16. Chen FK, Yeoh J, Rahman W et al. (2012): Topographic variation and interocular symmetry of macular choroidal thickness using enhanced depth imaging optical coherence tomography. Invest Ophthalmol Vis Sci., 53(2):975-985.

17. Gupta P, Saw SM, Cheung CY et al. (2015): Choroidal thickness and high myopia: a case-control study of young Chinese men in Singapore. Acta Ophthalmol., 93(7):585-92.

18. Ignacio F, Francisco L, Jay S et al. (2013): The relationship between axial length and choroidal thickness in eyes with high myopia. American Journal of Ophthalmology, 155(2): 314-319.

19. El-Shazly AA, Farweez YA, EISebaay ME et al. (2017): Correlation between choroidal thickness and degree of myopia assessed with enhanced depth imaging optical coherence tomography. Eur J Ophthalmol., 27(5):577-584.

20. Abdul W, Mousami M, Askari SN et al. (2015): Optical coherence tomography assisted macular thickness profile in high myopia Indian Journal of Basic and Applied Medical Research, 4(2): 159-166.

21. Minghui $\mathrm{Z}$, Qiang $\mathrm{W}$, Ping $\mathrm{H}$ u et al. (2015): Macular thickness assessed with optical coherence tomography in young Chinese myopic patients. J Ophthalmol., 2015: 715798.

22. Barrio-Barrio J, Noval S, Galdõs M et al. (2013): Multicenter Spanish study of spectral-domain optical coherence tomography in normal children. Acta Ophthalmologica., 91(1):56-63.

23. Al-Haddad C, Antonios R, Tamim H et al. (2014): Interocular symmetry in retinal and optic nerve parameters in children as measured by spectral domain optical coherence tomography. British Journal of Ophthalmology, 98(4):502-506.

24. Kim DY, Fingler J, Zawadzki RJ et al. (2012): Noninvasive imaging of the foveal avascular zone with high-speed, phase-variance optical coherence tomography. Invest Ophthalmol Vis Sci., 53:85-92.

25. Takase N, Nozaki M, Kato A et al. (2015): Enlargement of foveal avascular zone in diabetic eyes evaluated by en face optical coherence tomography angiography. Retina, 35:2377-2383.

26. Colin ST, Louis WL, Vernon SC et al. (2016): Optical coherence tomography angiography evaluation of the parafoveal vasculature and its relationship with ocular factors. Invest Ophthalmol Vis Sci., 57: 224- 234.

27. Bresnick GH, Condit R, Syrjala S et al. (1984): Abnormalities of the foveal avascular zone in diabetic retinopathy. Arch Ophthalmol., 102:1286-1293.

28. Chui TY, Van Nasdale DA, Elsner AE et al. (2014): The association between the foveal avascular zone and retinal thickness. Invest Ophthalmol Vis Sci., 55:6870-6877.

29. Dubis AM, Hansen BR, Cooper RF et al. (2012): Relationship between the foveal avascular zone and foveal pit morphology. Invest Ophthalmol Vis Sci., 53:1628- 1636.

30. Sung MS, Lee TH, Heo H et al. (2018): Association between optic nerve head deformation and retinal microvasculature in high myopia. Am J Ophthalmol., 188:81-90.

31. Al-Sheikh M, Phasukkijwatana N, Dolz-Marco R et al. (2017): Quantitative OCT angiography of the retinal microvasculature and the choriocapillaris in myopic eyes. Invest Ophthalmol Vis Sci., 58:20632069.

32. Min Li, Ye Yang, Hong $J$ et al. (2017): Retinal microvascular network and microcirculation assessments in high myopia. Am J Ophthalmol., 174: 56-67.

33. Coscas F, Glacet-Bernard A, Miere A et al. (2016): Optical Coherence Tomography Angiography in Retinal Vein Occlusion: Evaluation of Superficial and Deep Capillary Plexa. Am J Ophthalmol., 161:160-71.

34. Noma H, Funatsu H, Yamasaki M et al. (2008): Aqueous humour levels of cytokines are correlated to vitreous levels and severity of macular oedema in branch retinal vein occlusion. Eye (Lond), 22(1):4248.

35. Landa G, Rosen RB (2010): New patterns of retinal collateral circulation are exposed by a retinal functional imager (RFI). Br J Ophthalmol., 94(1):5458. 